\title{
Surgical blood loss: Should we keep estimating volume?
}

S. Jaramillo-Selman ${ }^{1}$, R. Navarro-Ripoll ${ }^{1}$, M. Montane-Muntane ${ }^{1}$, D. Capitan ${ }^{1}$, F. Aguilar ${ }^{2}$, A. Blasi-lbañez ${ }^{1,3}$.

${ }^{1}$ Department of Anesthesiology, Hospital Clinic de Barcelona, Universitat de Barcelona, Spain.

2 European Foundation for the Study of Chronic Liver Failure, Barcelona, Spain.

3 Institut d'Investigacions Biomèdiques Agusti Pi i Sunyer, Barcelona, Spain.

\section{BACKGROUND}

- Surgical blood loss is an essential parameter in anesthesiology, with relevant clinical and research applications.

- Regardless of the quantification method used, blood loss has always been expressed in a volume unit, ignoring the actual content.

- During surgery numerous variables constantly modify the composition of circulating blood. Blood loss is directly influenced by the composition of circulating blood. Therefore, quantification based exclusively on volume units could be misleading.

- Blood loss content has never been investigated.

- Main goal: To analyze the relation between the blood loss volume and the blood loss content, recovered during surgical procedures.

\section{MATERIALS AND METHODS}

- 45 consecutive patients undergoing robotic prostatectomy were studied.

- Blood loss was analyzed in the suction canister: hemoglobin concentration and volume were measured.

- Both blood loss volume and content were converted into values according to the patient characteristics:

- Blood loss volume: According to the patient's estimated blood volume (obtained by the ICSH formula).

- Blood loss Hb content: According to the patient's preoperative $\mathrm{Hb}$ concentration, and also according to the difference between the patient's pre- and postoperative $\mathrm{Hb}$ concentration.

- Other perioperative variables studied were: age, chronic hypertension, obesity, heart rate, MAP, surgical time, fluid administration, vasoactive drugs.

\section{- Analyses:}

- Correlation analysis between volumen and content (linear and nonlinear).

- Multiple correlation analysis (including perioperative variables).

- Regression analysis of previous EBL formulas.

- Univariate analysis of the influence of perioperative variables in the relationship between volume and content.
RESULTS
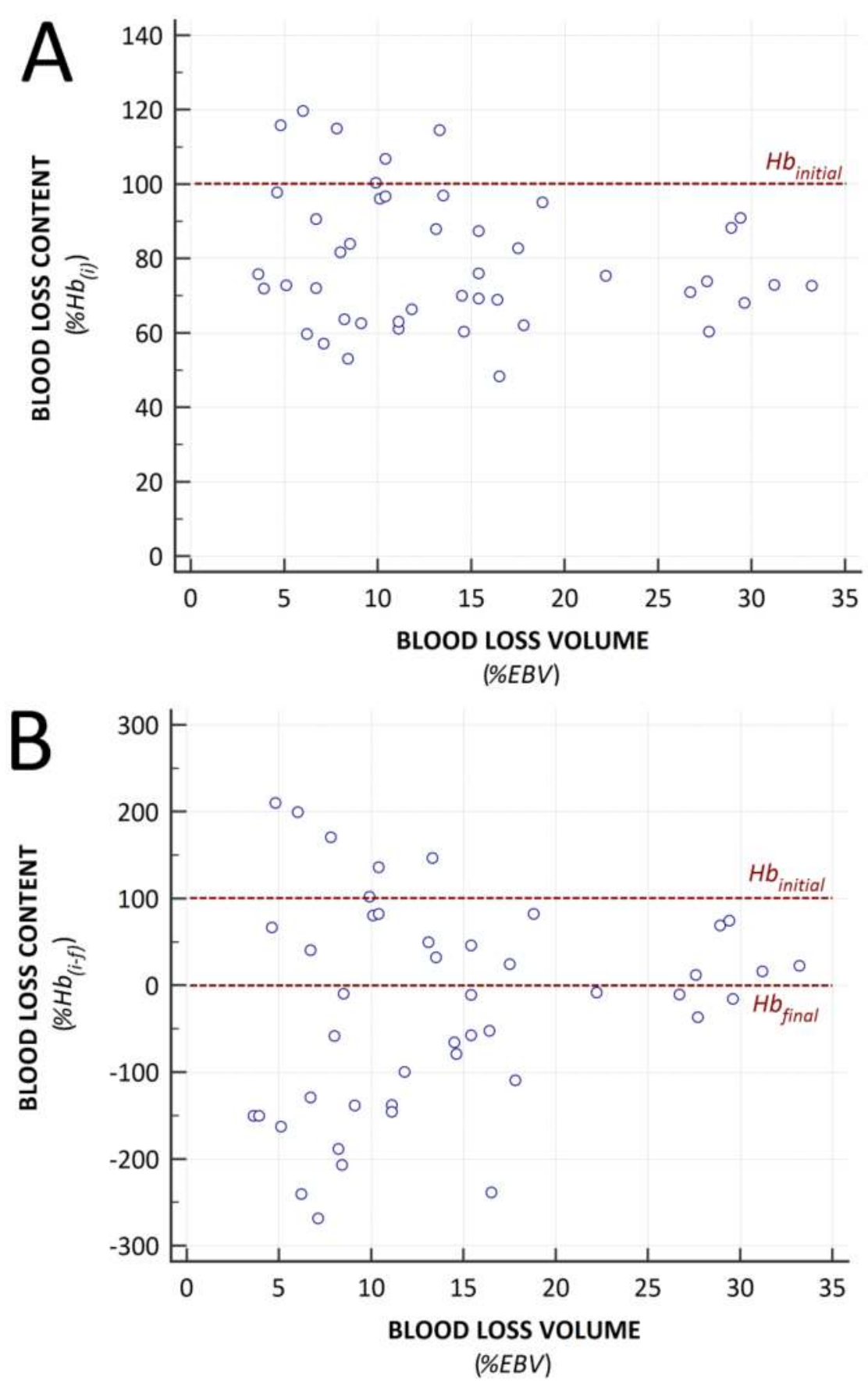

- Correlation analysis: Correlation coefficient between blood loss volume and content was found to be almost negligible in the linear $(r=0.108-$ $0.117, p<0.05)$ and nonlinear $(\rho=-0.142-0.139, p$ $<0.05$ ) analyses.

- Multiple correlation analysis: Perioperative variables studied did not explain the relationship between blood loss volume and content $(r=0.108-$ $0.117, p<0.05)$.

- Regression analysis of EBL formulas: The relationship could not either be explained by previous models $\left(r^{2} 0.008-0.039, p<0.05\right)$.

- Univariate analysis: The administration of vasoactive drugs (Ephedrine) during surgery was the only perioperative variable associated with an increased content of blood loss ( $p=0.045)$, without significant changes in blood loss volume $(p=$ 0.840).

\section{DISCUSSION AND CONCLUSIONS}

- The volume of blood loss does not correlate with its content.

- Our results suggest that volume is not a reliable method of quantifying blood loss.

- Further research using a standardized method capable of measuring both parameters would be desirable. 